\title{
Experiments and numerical modeling for the movement and resuspension of grains
}

\author{
Karina A. Valenzuela Aracena ${ }^{1}$, Jesica G. Benito ${ }^{1}$, Luc Oger $^{2}$, Rodolfo O. Uñac ${ }^{1}$, Irene Ippolito $^{3}$, Ana M. Vidales ${ }^{1, *}$ \\ ${ }^{1}$ INFAP, Dpto. de Física, CONICET, Universidad Nacional de San Luis, Ejército de los Andes 950, 5700 San Luis, Argentina \\ ${ }^{2}$ IPR, UMR UR1-CNRS 6251, Université de Rennes1, 263 Avenue du Général Leclerc, 35042 Rennes Cedex, France \\ ${ }^{3}$ Universidad de Buenos Aires, Facultad de Ingeniería, Grupo de Medios Porosos, Paseo Colón 850, 1063 C.A.B.A., Argentina
}

\begin{abstract}
We discuss two typical problems involved in particle resuspension phenomena. First, we analyze the onset of movement of a grain deposited on a surface prior to its possible resuspension. In this case, we look for the critical frequency and amplitude needed for starting the movement of the grain when the surface is perturbed by a sinusoidal vertical vibration. We compare results coming from our own experiments and DEM simulations. The agreement is good and allows evaluating the effect of the relative size of the grain on the initiation of movement. The second problem refers to the resuspension of grains, also deposited on a monolayer surface without interactions between them, due to the effect of aerodynamic forces. The detachment of grains as the wind intensity increases is studied through Monte Carlo simulations and the results are compared with experiments belonging to other authors. The role of the particle rolling mechanisms and the reduction factor due to roughness are discussed. Comparison of numerical and experimental data is promising.
\end{abstract}

\section{Introduction}

Industrial processes are full of examples where the manipulation of granular matter is implied. Energy is given to grains through different unitary operations such as vibration in conveyor belts, drilling operations, sieving and transport, among many others. Phenomena like bed fluidization, surface cleaning, or the reentrainment of particles to the atmosphere are based on the existence of a first incipient movement of grains to achieve the detachment of particles and their subsequent dynamic evolution [1-5]. The knowledge of the basic mechanisms causing the destabilization of a deposited particle on a horizontal surface is the key to proceed with its further dynamic description.

The study of the initiation of the movement of a particle when subjected to an external perturbation will of course depend on the scale of sizes involved in the problem. Nevertheless, we ask ourselves if some of the main aspects that are always present in the interaction among particles and surfaces prevail independently of the scale of the problem. One of these parameters will prove to be the roughness.

In the case of the starting of movement of a particle subjected to a continuous perturbation like vertical vibration or to wind bursts, it is mandatory to analyze the adhesion and capillary forces between the particle and the surface, the inertia of the particle when accelerated and its contact interaction with the particles belonging to the surface, especially in cases when rugosity is of the order of the size of the beads deposited on it $[6,7]$. This is not straightforward and the inclusion of all the parameters playing a role in the initiation of the particle movement becomes complicated.

In this work, we present part of our last results in studying the initiation of particle movement on a surface in two different scenarios. The first one deals with experiments and simulations (through a Discrete Elements Method, DEM) for spherical particles deposited on a rough surface that is subjected to a mechanical sinusoidal vibration. The idea behind is to find the critical amplitude-frequency pairs needed to initiate the movement of the deposited grains. The second involves simulations for modeling the resuspension of a monolayer of spherical particles deposited on a flat surface subjected to an air flow, and is developed based on a Monte Carlo (MC) method.

\section{Incipient movement of vibrated grains}

\subsection{Experimental procedure}

Experiments are performed using two different rough surfaces built by randomly gluing $500 \mu \mathrm{m}$ and $250 \mu \mathrm{m}$ glass spheres with epoxy on two smooth cylindrical surfaces, respectively. Each circle has $6 \mathrm{~cm}$ in diameter and a thin transparent wall around it to avoid the flow out of deposited particles. The surface is fixed to the arm of a mechanical wave driver whose generator can

\footnotetext{
* Corresponding author: avidales@unsl.edu.ar
} 
provide a sinusoidal oscillation at well controlled frequency and amplitude. Ten glass beads are randomly deposited over the rough surface and separated to avoid possible contacts or hits among them and/or with the lateral walls. Their diameters go from 1 to 4 millimetres.

The desired amplitude is selected (ranged from 0.7 to $4.2 \mathrm{~mm}$ ) in the generator and the platform is subjected to an oscillation starting from $0.1 \mathrm{~Hz}$ (approximately 0.63 $\mathrm{rad} / \mathrm{s})$. The frequency is increased in fixed steps at a rate of $0.1 \mathrm{~Hz}$ every second. With the help of a magnifying glass and a camera, we check for the incipient movement of particles and record the critical frequency at which at least half of the deposited spheres start to move from their original positions. Once the critical frequency is reached, the experimental run ends, and the oscillator is turned off. Several runs are performed to average data over 200 different positions.

A new value for the amplitude is chosen and the beads are redistributed again to start the searching of a new critical amplitude-frequency pair. Experiments using spheres with averaged diameters of 1.0, 2.0, 3.0 and $4.0 \mathrm{~mm}$ are performed separately.

\subsection{Simulation procedure}

We use a classical DEM model where the grains are simulated as spheres that can overlap each other, slip and rotate. We follow the two-dimensional formulation of Savage [8] and the three-dimensional extension used by Oger et al. [9]. Both normal and tangential forces develop at the contact between two spheres. In the case of the only presence of compressive forces, simulations represent dry, non-cohesive particle assemblies. The presence of tensile forces at the contacts models wet cohesive particles. In the present work, short range interactions can occur up to the breakage of the adhesive forces (i.e. breaking of a wetting film). No long range interactions are allowed. In order to simulate nonperfectly spherical particles and other local contact effects, rolling resistance is introduced [10] to represent the effects of particle shape on rolling.

Initially, the code creates the rough surface of glued glass spheres, i.e., immobile centres in the referential moving plate. Then, free glass spheres of a given size are randomly deposited on the rough surface for their future motion tracking. Finally, all the system is put under oscillation as in the experiments.

To resemble the experimental conditions, a disordered rough surface is created by a random $3 \mathrm{D}$ deposit of spheres with up to three layers. In all cases, all the spheres are sitting on a stable position under gravity with one contact in the case of the first layer, or up to three contacts for the other upper layers. Thus, a free sphere can sit on three underlying contacts distributed at different distances and heights on the rough surface.

\subsection{Comparison and analysis of results}

To ensure good statistics for data analysis, we check the dependence of the results on the number of free particles used to determine the different thresholds. A number of
200 particles (200 different sitting positions) seems to be a good approximation for both, experiments and simulations.

In Figures 1 and 2 we show the experimental results obtained for the critical frequencies, $\omega_{\mathrm{c}}$, needed to put into incipient movement the glass particles as the amplitude of the excitation is increased. The error bars for the amplitude are indicated and those for the critical frequency are of the order of the symbol size. The results correspond to the four different glass beads diameters. Figure 1 corresponds to a $250 \mu \mathrm{m}$ rough surface and Figure 2 corresponds to $500 \mu \mathrm{m}$. Thus, the size ratios used are, approximately, 4, 8, 12 and 16, in Figure 1, and 2, 4, 6 and 8, in Figure 2.

The points in each figures separate two regions in the plot: the upper part corresponds to the existence of movement of at least $50 \%$ of the spheres and the lower one to the absence of movement or to an incipient movement of less than $50 \%$ of the particles. Evidently, there is a decrease of the critical frequency as the amplitude increases. The trend can be approximated by an exponential decay, as displayed by the curves in both figures. Independently of their size and the size of the glued spheres, particles subjected to higher amplitudes of oscillation need a smaller critical frequency to initiate their movement. Only a weak dependence of the results on the size ratio is found.

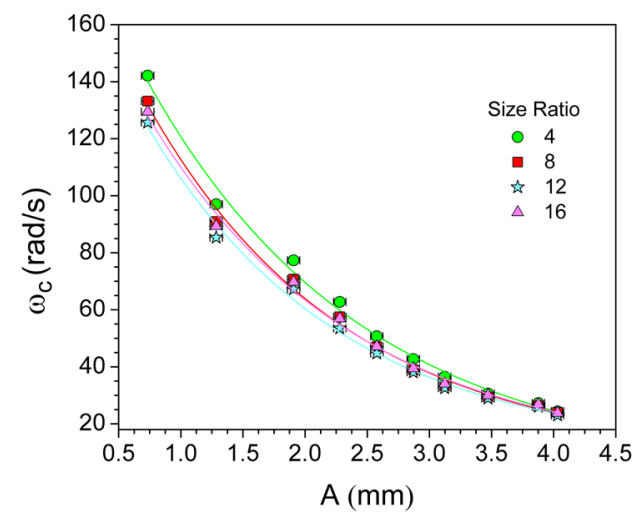

Fig. 1. Critical frequency as a function of the vibration amplitude for glass beads on $250 \mu \mathrm{m}$ glass surface.

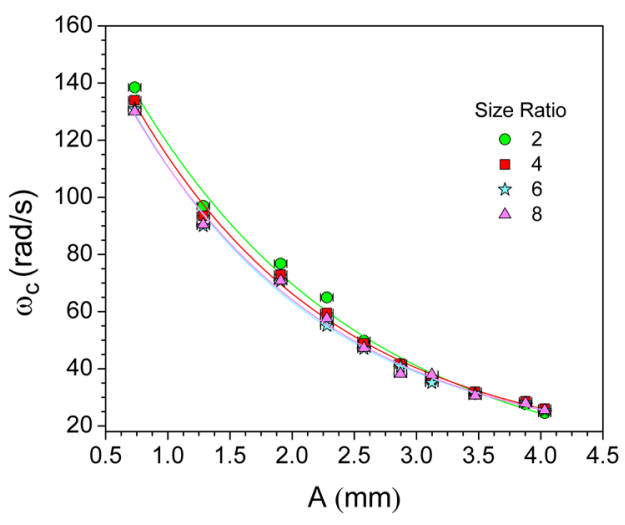

Fig. 2. Critical frequency as a function of the vibration amplitude for glass beads on $500 \mu \mathrm{m}$ glass surface. 
Figure 3 shows the comparison of the frequencyamplitude threshold evolution obtained with our numerical model with two sets of experimental results obtained with $500 \mu \mathrm{m}$ glued glass beads and similar conditions as in experiments. We observe the same exponential trend and the agreement is quite good for all the range of amplitudes studied. On the other hand, for $250 \mu \mathrm{m}$ glued glass beads, we can observe a considerable difference between experimental and numerical results although all the other parameters used are the same as in the previous comparison. The results are shown in Figure 4. The difference can be explained taking into account that, in simulations, the number of layers and the mean distance between spheres in these upper layers do not have the topological complexity of the surface generated through real glued spheres, as in the experiments.

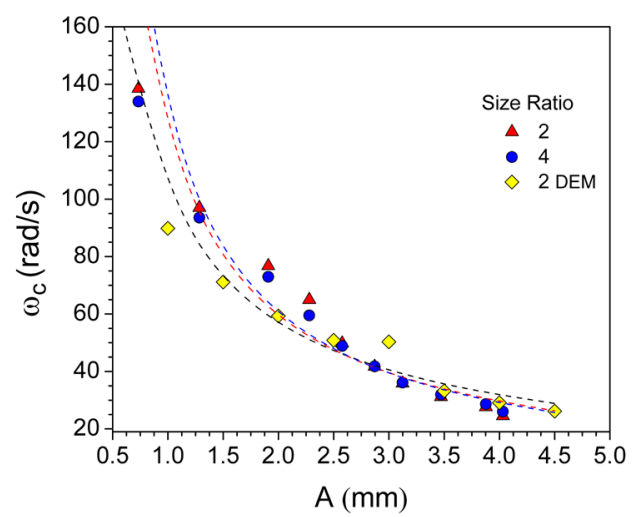

Fig. 3. Comparison between numerical and experimental results for a rough surface of $500 \mu \mathrm{m}$ glued glass beads.

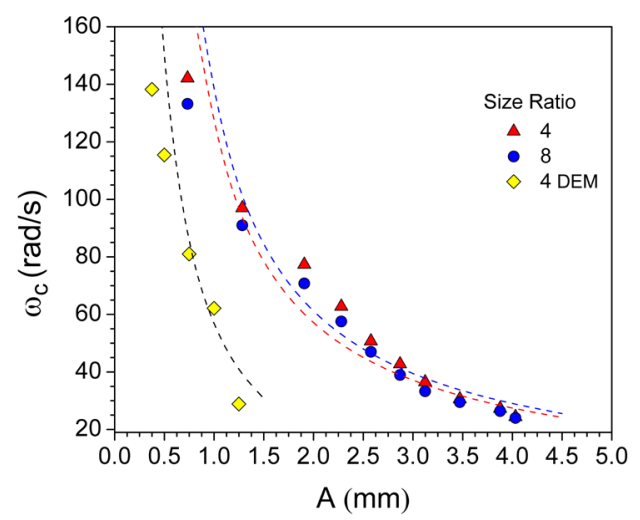

Fig. 4. Comparison between numerical and experimental results for a rough surface of $250 \mu \mathrm{m}$ glued glass beads.

The roughness of the surface created by the grains in the experiments is such that the characteristic length of this roughness is not dictated by the size of the constituting beads, but rather by the geometrical configuration of those beads in combination with the glue. Thus, the three-contact "tripods" formed by the free particles on top are topologically different from those arranged more horizontally in the simulations. In other words, the scale in the experimental surface structure hides the influence of the size of the constituting spheres. This is not the case in simulations where some level of order is still preserved because no glue effects are considered, thus preventing the formation of larger non horizontal "tripods" for the contacts of the free particles on the top of the surface. A more complex surface could be thought for future simulations for a deeper investigation of this hypothesis.

\section{Resuspension of grains}

\subsection{Simulation model}

A model for the resuspension of a monolayer of particles deposited on a flat surface is developed based on a Monte Carlo (MC) simulation. The distance between particles is not relevant in the present model since they do not interact with each other. Spherical particles are attached through an adhesion force that follows a lognormal distribution with a characteristic mean and dispersion, $\mu_{a}$ and $\sigma_{a}$, respectively. A turbulent air flow is assumed to be responsible for the resuspension of particles. A Gaussian distribution of aerodynamic forces is assumed with a mean $\mu_{h}$ and dispersion $\sigma_{h}$. The stochastic process used for resuspension is based on the evaluation of probabilities depending on the ratio between adhesion and aerodynamic forces and using a Metropolis function. Besides, the algorithm does not have to solve any integral equation, which implies quite short simulation times. Although simple, our model accounts for the main features of the resuspension flux observed experimentally and by other models $[11,12]$.

Typical experiments consist in measuring the resuspended amount of particles as a function of the friction velocity of the wind and especial attention is paid to the role that surface roughness has on the strength of the adhesion forces between particles and surface. Indeed, it has been proved that a strong reduction of adhesion is observed in the presence of a rough surface. In our simulations, this fact is taken into account through a reduction factor, $f_{F}$, that affect the strength of $\mu_{a}$ as follows

$$
\mu_{a}=F_{\mathrm{JKR}} / f_{r}=3 \pi \gamma R_{p} /\left(2 f_{r}\right)
$$

In this equation, $F_{\mathrm{JKR}}$ is the adhesion force estimated by the JKR theory due to Johnson, Kendall, and Roberts [13], i.e., the adhesion force between a particle of radius $R_{p}$ and the surface is given by $F_{\mathrm{JKR}}=3 \pi \gamma R_{p} / 2$, where $\gamma$ is the adhesive surface energy of the particle and substrate, and $f_{r}$ takes into account the contact geometry in a real surface which is usually characterized by a wide distribution of surface roughness.

We have already proved that results based on a force ratio balance [11] can qualitatively span a wide variety of expected behaviours. Nevertheless, the main mechanism observed in experiments is related to the rolling of particles as an incipient motion before succeeding in the re-entrainment to the fluid phase [1416]. Thus, the incorporation of the force moments in our MC model is developed, wherein the resuspension 
probabilities are evaluated from the balance between the adhesion and the aerodynamic moments acting on each particle. In the calculation of moments, the lever arm, $a_{0}$, corresponding to the adhesion force moment is crucial to understand that a particle is resuspended even when aerodynamic forces are less strong than adhesion ones. The value of $a_{0}$ depends on the deformation of the particle against the surface and on the presence of rugosity.

\subsection{Results and discussion}

Figure 5 shows the results for the fraction of resuspended particles for two different glass spheres diameters on a glass surface. The solid lines correspond to our simulation results. The open symbols correspond to several repetitions of experiments with $72 \mu \mathrm{m}$ glass spheres and filled symbols to repetitions with $32 \mu \mathrm{m}$ glass spheres due to Ibrahim and co-workers [16]. The reduction factor, $f_{r}$, is 200 and 43, respectively. Smaller particles present higher adhesion forces, thus the reduction factor is smaller. As said before, the presence of rugosity on the contacting surfaces makes adhesion to decrease and this effect is higher for larger particles. Nevertheless, the main reason why it is feasible destabilizing a particle is related to $a_{0}$, i.e., to the distance between the contacting points or "pivots" of the sphere sitting on the rough surface [12]. Thus, it is important to highlight that we have just varied the adhesive forces through the reduction factor, but, the lever arm $a_{0}$ in the moment calculation could be varied to obtain similar results. Both parameters allow taking into account roughness effects and, in fact, are connected to each other.

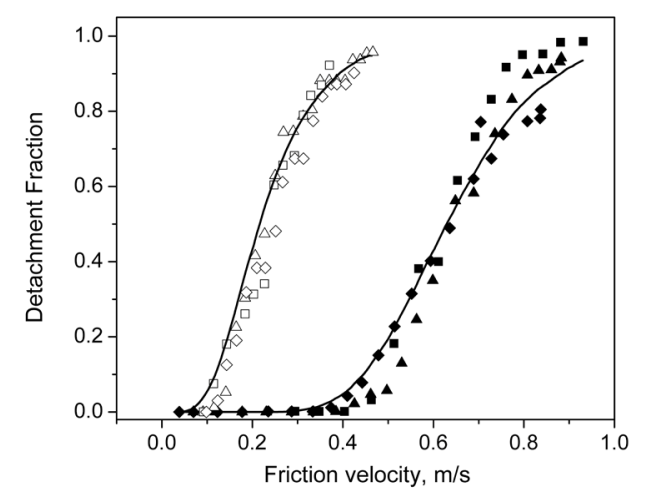

Fig. 5. Comparison between $\mathrm{MC}$ results and experimental data in [16]. Open symbols correspond to $72 \mu \mathrm{m}$ spheres and filled symbols to $32 \mu \mathrm{m}$.

\section{Conclusions}

We have explored two different scenarios to understand the incipient motion of grains subjected to different external perturbations. Although results belong to quite different scales (millimetre in the first case and micrometers in the second), they demonstrate that roughness is the key point to put in motion particles with different sizes. The rugosity intervening in the development of contacts between particles and surface is complex.

In simulations, attention should be paid when generating a rough surface through the arrangement of particles. The way in which free spheres are placed on the surface is crucial to evaluate their chance for movement. In a similar way, care should be taken when roughness factors are included in the description of adhesion forces and the role of the lever arm intervening in the rolling of the particles has to be taken into account in close relation to the rough nature of the surfaces in contact.

Roughness demonstrates to be the key parameter in the incipient movement and resuspension of particles and its role has still to be more deeply investigated.

The authors acknowledge financial support from ECOS-SudMINCyT project A15E03, CONICET-PIP No353 and CyTUNSL-PROICO No3-10114.

\section{References}

1. G. L. Dybwad, J. Appl. Phys. 58, 2789 (1985)

2. G. Ziskind, M. Fichman, C. Gutfinger, J. Aerosol Sci. 31, 703 (2000)

3. W. Theerachaisupakij, S. Matsusaka, M. Kataoka, H. Masuda, Adv. Pow. Technol. 13, 287 (2002)

4. Y. Mawatari, T. K. Y. Tatemoto, S. Uchida, K. Noda, Pow. Tech. 123, 69 (2002)

5. N. Tippayawong, I. Preechawuttipong, Aust. J. Basic Appl. Sci. 5356 (2011)

6. K. Hein, T. Hucke, M. Stintz, S. Ripperger, Part. Part. Syst. Char. 19, 269 (2002)

7. F. Soepyan, S. Cremaschi, B. McLaury, C. Sarica, H. Subramani, G. Kouba, H. Gao, Pow. Tech. 292, 272 (2016)

8. S. B. Savage, Disorder and granular media (D. Bideau Ed., North Holland, Amsterdam, 264, 1993).

9. L. Oger, I. Ippolito, A. Vidales, Gran. Matt. 9, 267 (2007)

10. J. Ai, J.-F. Chen, J. M. Rotter, J. Y. Ooi, Pow. Tech. 206, 269 (2011)

11. J.G. Benito, K.A. Valenzuela Aracena, R.O. Uñac, A.M. Vidales, I. Ippolito, J. Aerosol Sci. 79, 126 (2015)

12. J.G. Benito, R.O. Uñac, A.M. Vidales, I. Ippolito, J. Aerosol Sci. 100, 26 (2016)

13. K. Johnson, K. Kendall, A. Roberts, Proceedings of the Royal Society of London (Series A, Mathematical and Physical, 324, 301, 1971)

14. S. Fu, C. Chao, R. SO, W. Leung, J. Fluids Eng. 135, 041301 (2013)

15. I. Goldasteh, A. Goodarz, A. Ferro, J. Aerosol Sci. 66, 62 (2013)

16. A. Ibrahim, P. Dunn, R. Brach, J. Aerosol Sci. 29, 765 (2003) 